\title{
A large sample of objective prism quasar candidates
}

\author{
A. Iovino ${ }^{1}$, R. Clowes ${ }^{2}$ and P. Shaver ${ }^{3}$ \\ ${ }^{1}$ Osservatorio Astronomico di Brera, Via Brera, 28 I-20121 Milano, Italy \\ E-mail: iovino@brera.mi.astro.it \\ ${ }^{2}$ Centre for Astrophysics, University of Central Lancashire, Preston PR1 2HE, UK \\ E-mail: r.g.clowes@uclan.ac.uk \\ ${ }^{3}$ ESO, Karl-Schwarzschild-Strasse 2, D-85748 Garching bei Munchen, Germany \\ E-mail: pshaver@eso.org
}

Received January 2; accepted March 7, 1996

\begin{abstract}
The Automated Quasar Detection (AQD) technique has been applied to six connected fields near the South Galactic Pole. A comparison with the Véron \& Véron catalogue (1993) and the Cristiani et al. compilation (1995) shows that AQD re-discovered $\sim 75 \%$ of the known quasars with non-overlapping objective-prism spectra present in these fields ( $\sim 40 \%$ if only the high probability candidates are considered). A list of the 1592 high probability candidates is given, including the results of cross-correlation with $\mathrm{X}$-ray and radio catalogues. ${ }^{*}$
\end{abstract}

Key words: quasars: general — surveys

\section{Introduction}

This paper reports the results of the application of the Automated Quasar Detection (AQD) technique to six connected fields near the South Galactic Pole (total connected area $\sim 170 \mathrm{deg} .{ }^{2}$ ). AQD is a package developed to overcome the shortcomings of visual searches for quasars on objective prism plates (Clowes 1986a; Clowes et al. 1984; Clowes 1983). Briefly, objective prism plates, obtained at the UK Schmidt Telescope in Australia, are digitized by the COSMOS measuring machine (MacGillivray \& Stobie 1984) at the Royal Observatory in Edinburgh (ROE) and from the data the two-dimensional spectra corresponding to all objects are extracted for input to the AQD software. AQD then selects spectra according to various options (typically emission lines, absorption lines, spectral discontinuities, ultraviolet excess and red excess), within specified numerical limits. In this paper, emission lines and ultraviolet excess only have been used.

This paper is divided into two parts. The first gives details of the application of AQD to our fields and of the grading procedure chosen to select the best candidates. The second presents the results of cross-correlations of the best candidates with catalogues of known quasars and radio sources. The large database obtained from this survey is

Send offprint requests to: A. Iovino

${ }^{\star}$ Table 3 is only available in electronic form at the CDS via anonymous ftp 130.79.128.5 valuable for many areas of quasar research, e.g. clustering of quasars and study of close pairs for common absorption systems. 14.Sep.'90: Demo-Vs.

\section{Candidate selection}

Table 1 shows the field centres and numbers of the plates used.

Table 1.

\begin{tabular}{lllll}
\hline $\begin{array}{l}\text { ESO/SERC } \\
\text { field }\end{array}$ & \multicolumn{2}{l}{ Field Centre (1950) } & $\begin{array}{l}\text { Direct } \\
\text { Plate }\end{array}$ & $\begin{array}{l}\text { Prism } \\
\text { Plate }\end{array}$ \\
\hline 294 & 002600 & -400000 & J3351 & UJ5412P \\
295 & 005200 & -400000 & J2712 & UJ6536P \\
296 & 011800 & -400000 & J3524 & UJ5406P \\
297 & 014400 & -400000 & J3593 & UJ4514P \\
351 & 004800 & -350000 & J8046 & UJ6528P \\
411 & $004600-300000$ & J4606 & UJ7307P \\
\hline
\end{tabular}

Each prism plate was obtained with unfiltered IIIa-J emulsion, giving a wavelength coverage from 3200 to $5380 \AA$. Each direct plate was obtained with the IIIa-J emulsion plus the GG395 filter, corresponding to the $B_{\text {J }}$ photometric band (3950 to $5380 \AA$ ). For each field the central $\sim 28.3$ deg. ${ }^{2}$ (both of the direct and objective prism plate) are 
digitized using COSMOS, with $16 \mu \mathrm{m}$ spot size, $16 \mu \mathrm{m}$ pixel size and 14 bit digitisation.

The first stages of the AQD process involve only the direct plates. First, the images are classified as star-like or galaxy-like (for a discussion on the star/galaxy classification problems, see MacGillivray \& Stobie 1984). Images are then further classified according to whether they would be geometrically overlapped on the corresponding prism plate. An image is classified as a geometrical overlap if a second image occurs within \pm 70 pixels in the $x-$ direction (the dispersion direction) and \pm 3 pixels in the $y$-direction (the scale of our plates is $67.14 \mathrm{arcsec} / \mathrm{mm}$, i.e. $\sim 1 \mathrm{arcsec} /$ pixel). The direct plates are also used to obtain the celestial coordinates of each objects, with a precision of the order of $\sim 1$ arcsecond.

On the prism plates the spectra having an intensity greater than $10 \%$ of the sky background and a connected area greater than 30 pixels are extracted. Using this first set of two-dimensional spectra, the coordinate transformation from direct to prism plates is determined, and then used to extract all spectra, corresponding to images with $B \leq 21$ on the direct plates, in spectrum blocks of $128 \times 8$ pixels. Approximately $\sim 370.000$ spectra were extracted, of which $\sim 64.500$ were overlapped, i.e. $\sim 17.4 \%$. The extracted spectra were then processed by the AQD software, which, compared with visual searches on prism plates, has the great advantage that the selection criteria and, consequently, the physical selection effects, are known, predefined, and rigidly maintained. AQD can select quasar candidates by emission lines, absorption lines, spectral discontinuities, ultraviolet excess and red excess. For this survey we have used only the two most productive criteria: emission lines and ultraviolet excess (see Clowes et al. 1984; Clowes 1986b, for details). The criterion used to recognize an emission line is that its peak exceeds a $\mathrm{S} / \mathrm{N}$ of 8 with respect to the continuum. For selection by ultraviolet excess two broad band filters were defined for the ranges $3486-3815 \AA$ and 4170 - $4659 \AA$ : magnitudes were assigned in these bands, and candidates were selected when the ultraviolet excess in these bands exceed a specified limit (0.30 for all the fields, except 351 and 295, for which it was 0.4$)$. Note that the selection by ultraviolet excess uses the continuum and so avoids biases from spectral lines. The AQD selection also checks for objects classified as geometrical overlaps and objects that are too faint, saturated or otherwise flawed, and rejects them. This way we obtained a total of 16.784 quasar candidates in our 6 fields, up to a limiting magnitude of $B_{\mathrm{J}} \sim 20.5$ for the best plates. Table 2 lists, for each field, the number of spectra extracted, the number of overlapping spectra and the number of quasar candidates. table

The precise magnitude limit for each plate is unknown, because of a lack of external data for calibration; therefore no significance should be attached to the variations in surface density from field to field. Of course, not all the
Table 2.

\begin{tabular}{llll}
\hline Field & $\begin{array}{l}\text { Extracted } \\
\text { spectra }\end{array}$ & $\begin{array}{l}\text { Overlapping } \\
\text { spectra }\end{array}$ & $\begin{array}{l}\text { Quasar } \\
\text { candidates }\end{array}$ \\
\hline 294 & 55945 & 8211 & 1907 \\
295 & 62592 & 10549 & 3505 \\
296 & 57320 & 8559 & 2723 \\
297 & 60179 & 9604 & 3431 \\
351 & 75695 & 18021 & 2624 \\
411 & 58199 & 9490 & 2594 \\
& & & \\
Total & 369930 & 64434 & 16784 \\
\hline
\end{tabular}

objects that satisfy the AQD selection criteria are quasars, and, ideally, spectroscopy should be used to identify exactly which objects are quasars. To minimize contamination in the final list, we have devised a grading scheme to select the highest probability candidates from the sample extracted.

Our grading scheme is a very simple one, in which points are awarded according to quasar-like properties, so that the sum of the points for a given candidate is directly related to the probability of its being a quasar. The grading scheme adopted is the following:

0 - if the candidate is classified as galaxy-like by COSMOS on the direct plate,

+1 - if the candidate is classified as star-like by COSMOS on the direct plate,

$+1-$ if the candidate has ultraviolet excess,

+1 - if the candidate has emission lines with (line/continuum intensity) $\geq 0.2$ (this was empirically found to be a good criterion to reject obvious stellar contaminants),

-1 - if the candidate is bright $\left(m_{\text {COSMOS }} \leq 2.2\right)$ and has a 'stellar feature' ('stellar feature' is defined as the presence of an 'emission' line detected around $\lambda \sim$ $4100-4200 \AA$, ie. most probably a spurious line, mimicked by the two absorption features $H-K$ and $G$-band in a star spectrum),

- lastly, if the candidate has an emission line, a term is added quantifying the strength of the line (this term is defined in such a way that it is always less than 1).

We selected a high probability subset of quasar candidates as those having grade $\geq 2.3$ (except for fields 411 and 294, for which this limit was lowered to 2.2 to take into account the fact that these two plates are of lower quality).

For example, a candidate classified as star-like, having ultraviolet excess and an emission line with (line/continuum intensity) $\geq 0.2$, is accepted in the high probability subset irrespective of the strength of the emission line (its grade is always $\geq 3$ ), while a candidate classified as star-like, 
having an emission line with (line/continuum intensity) $\geq$ 0.2 , and no ultraviolet excess is accepted in the high probability subset only if the emission line is strong enough that the term quantifying the strength of the strongest line is greater than 0.3 (or 0.2 for fields 411 and 294).

\section{Best candidates and cross correlation with known catalogues}

Table 3 lists the 1592 high probability quasar candidates for all five fields. For each candidate we give RA, DEC, both 1950 ;

the prism instrumental magnitude as measured by COSMOS (on the prism plate, in the wavelength range $\left.4500-5000 \AA, m_{\mathrm{COSMOS}}\right)$; an indicative magnitude in the $V$ band $\left(V_{\text {COSMOS }}\right)$, obtained using the known quasars in each field to estimate a rough zero point for COSMOS prism plate magnitudes; a tentative redshift, assigned assuming that the strongest line visible on the spectrum was Ly $\alpha$ and the grade assigned according to the method described above. Furthermore, for each candidate there is a column of comments. A? in this column indicates a possible overlapping spectrum (not recognized as such by the criterion used to define a spectrum as an overlap) or a possible star, while a * indicates a good quality candidate (judged from the appearance of the 1-D spectrum). In the same column, a $\mathrm{Q}$ indicates a known quasar, an $\mathrm{R}$ a known radio source and an $\mathrm{X}$ a known ROSAT $\mathrm{X}-$ ray source. To obtain this information we cross-correlated the list of AQD candidates with the Véron \& Véron catalogue of quasars (1993), with the Cristiani et al. ESO key-programme quasars (1995), with the Parkes and PMN catalogue of radio sources (Griffith \& Wright 1993; Griffith et al. 1994; Wright et al. 1994) and with the WGACAT catalogue of sources in the pointed ROSAT observations (White et al. 1994). Given the large error boxes for the position of the radio and X-ray sources (up to $60^{\prime \prime}$ ), the $\mathrm{X}$ and $\mathrm{R}$ represent only probable identifications. Table 3 is only available in electronic form at the CDS via anonymous ftp 130.79.128.5.

It is interesting to examine the statistics obtained from the cross-correlation with known quasars. AQD detected 456 out of the 706 quasars brighter than $V=20.5$ known in the literature in our fields (20.5 in $B_{\mathrm{J}}$ is, approximately, the magnitude limit for our best fields). Part of the incompleteness is accounted for by the rejection of overlapping spectra, which makes our database incomplete by a fraction $\sim 17.4 \%$. This percentage translates into $\sim 110$ quasars lost because of overlapping spectra. We therefore estimate the true fraction of quasar re-discovered by AQD to be at least $\sim 75 \%$ of those existing in the literature, and this is just a lower limit, as the magnitude limit of 20.5 holds only for the very best plates.

Figure 1 shows, for each of the known quasars in our fields re-discovered by AQD, the histogram of the difference between $\mathrm{V}$ magnitudes (when quoted in the literature) and
$m_{\mathrm{COSMOS}}$ magnitudes, measured on the prism plate, in the wavelength range $4500-5000 \AA$.

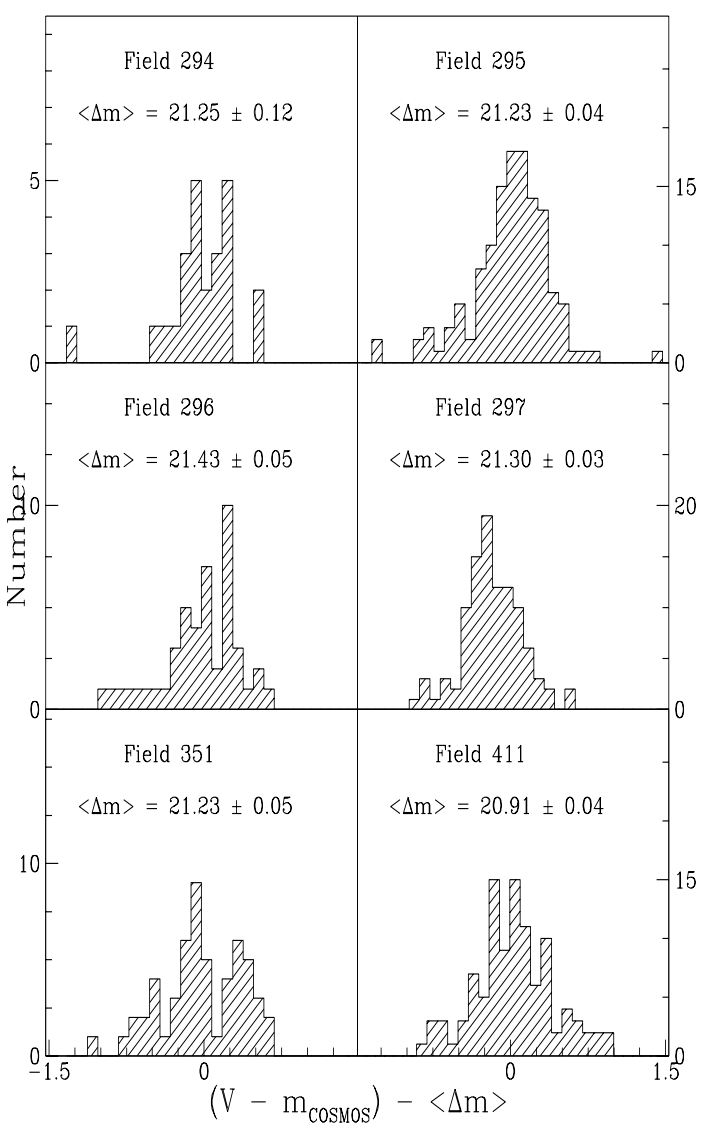

Fig. 1. Histogram, for each of the known quasars in our fields, of $\Delta m$, i.e. $V$ magnitudes as published in the literature minus $m_{\text {Cosmos }}$ magnitudes. The $\Delta m$ mean value and its sigma for each field are quoted

It is possible to determine in this way a zero point for each field, that, as Fig. 1 shows, is quite accurate. This zero point has been used to determine the $V_{\mathrm{COSMOS}}$ magnitudes shown in Table 3.

Figure 2 shows, for all the quasar candidates listed in Table 3 , the histogram of the estimated $V_{\text {Cosmos }}$ magnitudes. The incompleteness is obvious in the last magnitude bins.

Figure 3 shows the redshift distribution of all the quasars with published redshifts in our fields and of those detected by AQD (shaded histogram). A Kolmogorov-Smirnov test applied to the two redshift distributions (restricted to the range 0 to 2.6, to avoid effects due to the incompleteness in magnitude at higher redshifts) shows that the probability of the difference between the two distributions being greater than that observed is $\sim 70 \%$. This comparison shows that AQD has been successful in finding quasars over a wide range of redshifts, with no obvious selection 


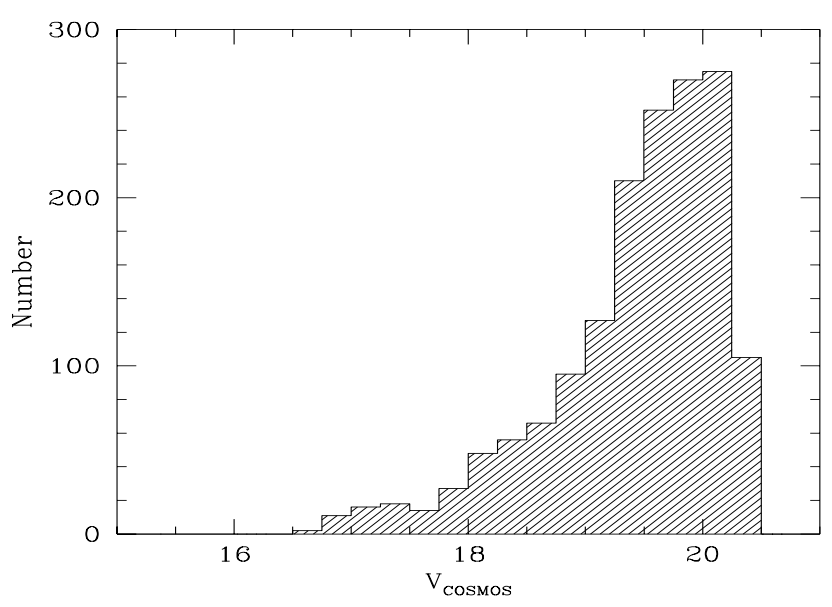

Fig. 2. Histogram, for the sample of high probability candidates, of the estimated $V_{\text {COSMOS }}$

bias relative to the Véron \& Véron catalogue and the Cristiani et al. quasars list.

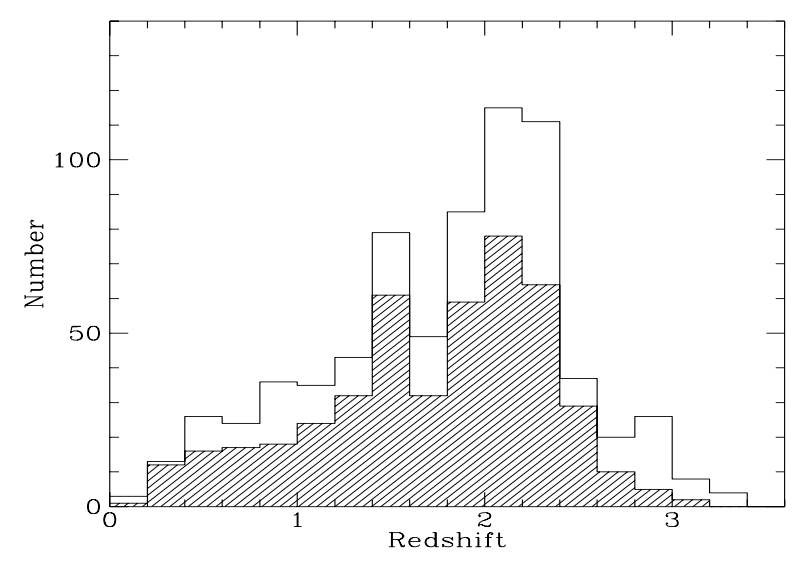

Fig. 3. Histogram of the redshift distribution for the known quasars in our fields and those re-discovered by AQD (shaded)

Of course, there may be selection biases in the Véron \& Véron catalogue itself, which vary depending on the survey techniques which happen to have been used in different areas of sky. In the region covered by the AQD survey, the Véron \& Véron catalogue is dominated by the objective prism surveys of Drinkwater (1987), Kunth \& Sargent (1986), Morris et al. (1991), and the UVX colour survey of Boyle et al. (1990). The good agreement with the latter survey (24 quasars found out of 61 present in

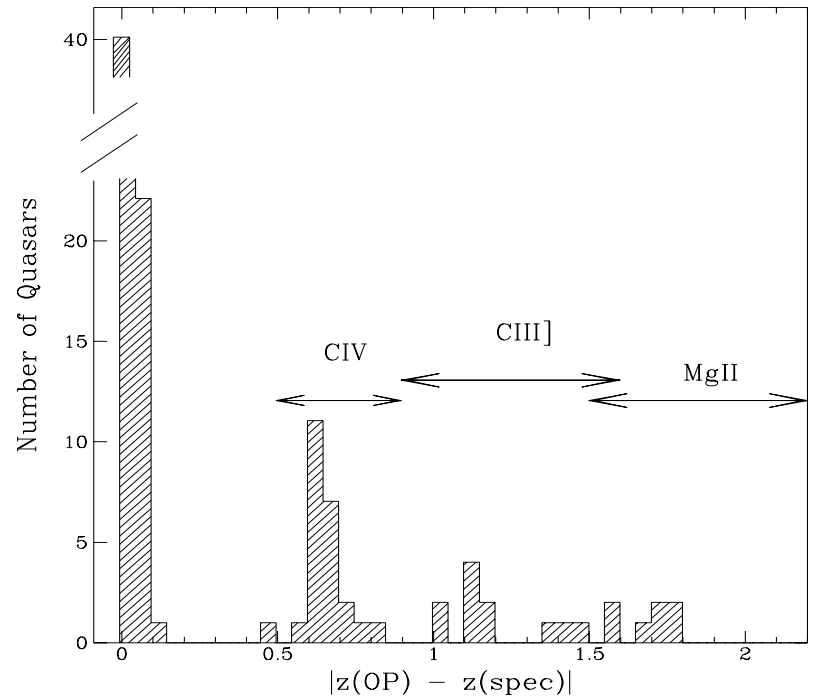

Fig. 4. Distribution, for each of the known quasars in the high probability subset, of the difference between the redshift as estimated from $\mathrm{AQD}(z(\mathrm{OP}))$, and that quoted in the literature $(z(\mathrm{spec}))$

our fields), shows that AQD has been successful in finding UVX quasars, and so is not just restricted to strongline objects. This is confirmed also by the good agreement with the Cristiani et al. survey, where bright quasars are selected using ultraviolet excess: out of 72 new quasars present in our fields, 46 were detected by AQD.

The grading system is successful in picking up the best quasar candidates: out of the 456 quasars detected by AQD, 246 are in the high probability subset. That is, $54 \%$ of the known quasars in our fields are in the high grade subset that comprises less than $1 \%$ of the total sample extracted by AQD. Even if we consider only the high probability candidates the fraction of quasar re-discovered by AQD is still as high as $\sim 40 \%$.

For the 246 quasars that are in the high probability subset, we can compare the difference between the redshift published in the literature and that estimated by AQD from the objective prism plate. Before doing this comparison, we rejected from the list of common quasars those having in the literature only a slitless spectrum, or a redshift quoted to fewer than 2 decimal digits, being left with 101 quasars. The results of the comparison are shown in Figs. 4 and 5. Figure 4 shows the absolute value of the difference between AQD redshifts and redshifts published in the literature, for the quasars in the high probability subset. The peak around zero corresponds to those quasars for which the most prominent line in the objective prism spectrum is a Ly $\alpha$ line, and therefore the redshift has been correctly estimated by AQD (notice that only $\sim 40 \%$ of the quasars in the high probability subset are non Ly $\alpha$ ). 
The other clumps at different positions correspond, respectively, to cases in which a line different from $\operatorname{Ly} \alpha$ was the most prominent one in the objective prism spectrum.

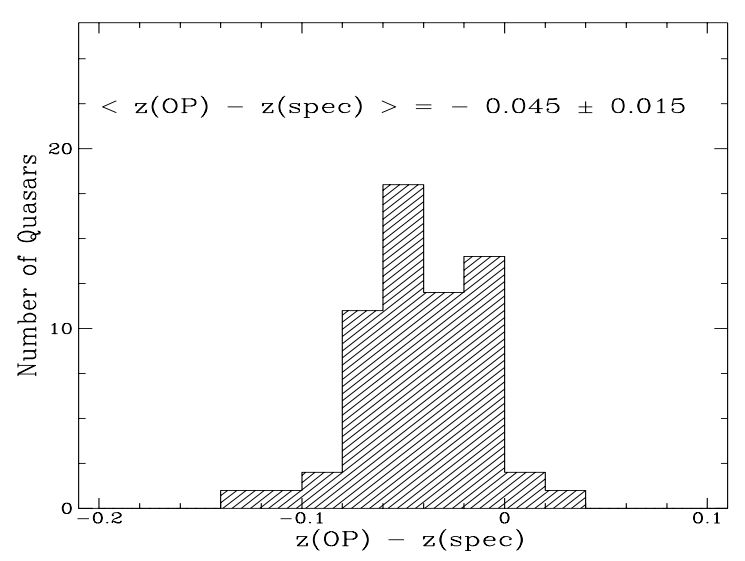

Fig. 5. Histogram, for each of the known Ly $\alpha$ quasars of the high probability subset, of the difference between the redshift as estimated from $\mathrm{AQD}(z(\mathrm{OP}))$ and that quoted in the literature $(z(\mathrm{spec}))$

Figure 5 shows in detail the value of the difference between AQD redshifts and redshifts published in the literature, for the Ly $\alpha$ quasars. After a correction of 0.045 (most probably due to a small systematic error in the wavelength calibration), the sigma of the difference between the two redshift estimate is only 0.015 (note that the redshifts listed in Table 3 are the original measured values, i.e. not corrected for this shift of 0.045). The surface density of quasar candidates in the high probability subset is $\sim 10 \mathrm{deg} .^{-2}$ for the best fields, which compares well with a surface density of $\sim 30$ deg. $^{-2}$ as reported in the literature for quasars brighter than $V \sim 20.5$ (Hartwick \& Schade 1990). This suggests that the high probability subset contains $\sim 30 \%$ of the quasars to be expected in the corresponding area.

Acknowledgements. The staff of the COSMOS Unit at ROE and of the UK Schmidt Telescope Unit at ROE and Coonabaraban are thanked for their help. Most of the data processing used the STARLINK facilities at ROE. We would also like to thank the referee, Dr. P. Véron, for the useful comments and suggestions.

\section{References}

Boyle B.J., Fong R., Shanks T., Peterson B.A., 1990, MNRAS 243, 1

Clowes R.G., 1983, in: Quasars and Gravitational Lenses, 24th Liège Astrophysical Colloquium, p. 26

Clowes R.G., Cooke J.A., Beard S.M., 1984, MNRAS 207, 99

Clowes R.G., 1986a, Mitteilungen der Astron. Ges. 67, 174

Clowes R.G., 1986b, MNRAS 218, 139

Cristiani S., La Franca F., Andreani P., et al., 1995, A\&AS 112,347

Drinkwater M.J., 1987, Quasar Clustering on Large Scale, PhD Thesis, Cambridge

Griffith M.R., Wright A.E., 1993, AJ 105, 1666

Griffith M.R., Wright A.E., Burke B.F., Ekers R. D., 1994, AJ 90, 179

Hartwick F.D.A., Schade D., 1990, ARA\&A 28, 437

Kunth S.L., Sargent W.L.W., 1986, AJ 91, 761

MacGillivray H.T., Stobie R.S., 1984, Vistas Astr. 27, 433

Morris S.L., Weymann R.J., Anderson S.F., et al., 1991, AJ 102,1627

Véron-Cetty M.-P., Véron P., 1993, ESO Scientific Report 13

White N., Giommi P., Angelini L., 1994, IAU Circ. 6100

Wright A.E., Griffith M.R., Burke B.F., Ekers R.D., 1994, ApJS 91, 111 